\title{
س
}

$>\mathrm{DE}$

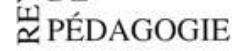

\author{
Recherches en éducation
}

166 | janvier-mars 2009

Varia

\section{La mise en incitation des enseignants : solution théorique ou réponse pragmatique?}

Giving incentives to teachers: theoretical solution or pragmatic answer?

Poner en incitación a los docentes: ¿Solución teórica o respuesta pragmática?

Steueranreiz bei der Lehrerschaft: theoretische Lösung oder pragmatische Antwort?

\section{Françoise Larré}

\section{(2) OpenEdition}

\section{Journals}

Édition électronique

URL : http://journals.openedition.org/rfp/1101

DOI : 10.4000/rfp. 1101

ISSN : 2105-2913

Éditeur

ENS Éditions

\section{Édition imprimée}

Date de publication : 1 mars 2009

Pagination : 27-43

ISBN : 978-2-7342-1152-5

ISSN : 0556-7807

Référence électronique

Françoise Larré, «La mise en incitation des enseignants : solution théorique ou réponse pragmatique? », Revue française de pédagogie [En ligne], 166 | janvier-mars 2009, mis en ligne le 01 mars 2013, consulté le 19 avril 2019. URL : http://journals.openedition.org/rfp/1101 ; DOI : 10.4000/ rfp.1101 


\section{La mise en incitation des enseignants : solution théorique ou réponse pragmatique? \\ Françoise Larré}

Les enseignants sont-ils réceptifs aux incitations monétaires ? De nombreuses études montrent, conformément aux modèles théoriques, que ces incitations ont des implications directes sur le comportement des enseignants. Mais elles montrent également que le changement de comportement des enseignants ne s'effectue pas toujours dans le sens attendu ou désiré. À partir de ce constat et sur la base d'une revue de la littérature sur les incitations, nous proposons d'examiner la portée pragmatique de la mise en incitation des enseignants d'un quadruple point de vue : technique, organisationnel, culturel et économique.

Descripteurs (TESE) : enseignant, ressources humaines, incitation financière, enseignement public.

\section{INTRODUCTION}

La légitimité de la logique doctrinale dont l'État et les services publics, notamment le secteur éducatif non marchand, étaient traditionnellement porteurs, est depuis quelques dizaines d'années fortement contestée. Les remises en cause répétées des objectifs, des méthodes et des résultats des services publics ont préparé les esprits à la nécessité de s'inscrire dans une recherche de performance, inspirée des valeurs économiques du secteur marchand. C'est donc selon une logique d'efficience que progressivement se diffusent au secteur non marchand certaines formes de logique qui furent longtemps l'apanage du secteur marchand.
Ainsi en est-il du secteur éducatif (Mingat \& Morlaix, 2001) qui, dans de nombreux pays, a fait l'objet de réformes visant en particulier à évaluer les établissements et les personnels et à tenter d'orienter leurs actions à travers l'utilisation de mécanismes incitatifs. Les enseignants des pays anglo-saxons, d'Amérique latine, d'Europe du Nord ont ainsi expérimenté les incitations sous diverses formes et avec plus ou moins de bonheur. Très récemment, en France, la loi organique relative aux lois de finances (abrégée en LOLF) a ouvert la voie à l'introduction d'une rémunération incitative dans les services publics et le rapport du ministre sur la situation morale et matérielle des professeurs en France propose que " les professeurs dont les élèves auront progressé ou réussi leurs examens ou certifications, les professeurs qui auront 
conduit avec succès à leur terme des projets pédagogiques ou éducatifs puissent bénéficier de primes " (Darcos, 2006, p. 21). C'est ainsi que la question des mécanismes incitatifs, de leurs modalités, de leur acceptabilité et de leur efficacité, resurgit à nouveau.

Les incitations, en général, et les incitations envers les enseignants en particulier ont en effet déjà fait l'objet de nombreux débats, aux plans éducatif et politique. À l'évidence, "les incitations comptent, pour le meilleur et pour le pire » (Prendergast, 1999) au sens où elles ont des implications directes sur les caractéristiques et le comportement des enseignants. Cependant, ce qui est beaucoup moins clair, c'est la façon dont les incitations fonctionnent et les conditions sous lesquelles elles créent les types de changements désirés (Clotfelter et al., 2004 ; Dee \& Keys, 2004 ; Ebberts et al., 2002 ; Hanushek, 2003 ; Jacob \& Levitt, 2003a ; Koretz, 2002 ; Lavy, 2002, 2003, 2004 ; Prendergast, 1999).

Cet article fournit un rapide examen de la littérature traitant des incitations monétaires individuelles destinées aux enseignants. Bien qu'il existe une " autre " littérature traitant des incitations individuelles non monétaires et des incitations collectives dont la mise en œuvre semble ouvrir des pistes de réflexion prometteuses, nous nous en tiendrons ici aux incitations monétaires individuelles. Parce qu'elles resurgissent de façon récurrente dans le discours politique comme solution aux "difficultés de l'école ", il semble plus que jamais nécessaire d'examiner les divers arguments et résultats concernant leur mise en œuvre auprès des enseignants, en relation avec la nature de l'activité d'enseignement et avec les caractéristiques et comportements des enseignants.

Le point de départ de cet article sera la présentation de la théorie de l'agence, dite aussi théorie « principal-agent ». Cette théorie est aujourd'hui largement utilisée pour traiter de l'amélioration de la performance du secteur public et des nouveaux modes de régulation sur les marchés jusque-là gérés par la fonction publique d'État, comme les marchés de l'énergie, de l'eau, des transports ou des télécommunications (Crampes \& Laffont, 1995 ; Laffont \& Tirole, 1993). À l'occasion de son application à la régulation du secteur public, la théorie de l'agence fut rebaptisée théorie des incitations et c'est dans ce cadre que s'inscrit le principe de mise en incitation des enseignants (tout comme d'ailleurs le principe d'autonomie des établissements scolaires). Si la perspective théorique d'une telle mise en incitation semble aller de soi, sa mise en œuvre pratique mérite d'être examinée de façon plus approfondie. Dans la suite de l'article, nous proposons donc de confronter cet outil théorique à diverses dynamiques concrètes afin d'en mesurer la portée pragmatique. Au plan technique, nous examinerons la question de l'évaluation et de sa faisabilité lorsqu'elle concerne des enseignants, travailleurs du savoir qui exercent une activité de service. Au plan organisationnel, nous examinerons dans quelle mesure les schémas incitatifs sont adaptés aux caractéristiques de l'activité de travail des enseignants. Au plan culturel, nous examinerons les motivations des enseignants et les risques associés aux structures incitatives, notamment les dysfonctionnements de comportement, en réponse au système d'incitation. Enfin, au plan économique, nous examinerons l'efficience des mécanismes incitatifs, en particulier l'équilibre entre les coûts de la mise en incitation et ses bénéfices à court et à long terme.

\section{LE PRINCIPE DE LA MISE EN INCITATION DES ENSEIGNANTS}

La question de la mise en incitation des enseignants est réapparue récemment avec les développements de la "New economics of personnel " (Lazear, 1998), qui consistent en l'application de la théorie des incitations à la gestion des ressources humaines. L'objectif est d'identifier les formes contractuelles incitatives optimales en matière de gestion des salariés. Dans ces contributions, la question des incitations et des formes contractuelles incitatives est abordée de façon très spécifique puisque les contrats sont circonscrits à un système de rémunération particulier, censé inciter l'agent à augmenter ses efforts. Les systèmes de paiement (Lazear, 1986), de promotion (Lazear \& Rosen, 1981) et d'évaluation (Baker et al., 1994) sont analysés comme des dispositifs de compensation monétaire de l'effort.

Le cadre d'analyse sur lequel s'appuient les auteurs est celui de la théorie de l'agence. La théorie de l'agence s'intéresse à la conception de contrats bilatéraux, lorsque des individus mûs par leur intérêt personnel concluent un arrangement contractuel implicite ou explicite. Le modèle d'agence le plus simple suppose qu'un individu ou une organisation (dit " principal ») délègue à un autre individu ou organisation (dit " agent ") la responsabilité de choisir ou d'exécuter une action, ce qui implique la délégation d'une partie de l'autorité de prise de décision à l'agent. La définition généralement retenue est celle 
proposée par Michael Jensen \& William Meckling (1976, p. 308) : "Nous définissons une relation d'agence comme un contrat dans lequel une (ou plusieurs) personne(s) a (ont) recours aux services d'une autre personne pour accomplir en son nom une tâche quelconque, impliquant une délégation de nature décisionnelle à l'agent ". Cette délégation est motivée par le fait que l'agent a des capacités, des qualifications, des compétences et/ou des savoirs particuliers que n'a pas le principal. Le principal estime donc que l'agent est plus apte que lui à réaliser l'action déléguée. L'agent est rémunéré par le principal.

La caractéristique essentielle d'une relation d'agence est l'asymétrie de l'information : asymétrie liée aux différences de capacités ou de compétences du principal et de l'agent, mais aussi asymétrie qui découle de la non-observabilité par le principal des caractéristiques et des efforts de l'agent. En effet, l'agent connaît ses propres caractéristiques et/ou l'action choisie mais le principal est incapable de savoir, à partir de la seule observation des résultats, quelle combinaison de caractéristiques, d'actions et d'éléments contextuels a été mise en œuvre par l'agent. Le principal peut donc se trouver confronté à une incertitude forte concernant le comportement de l'agent, qu'il peut difficilement apprécier ou dont le coût d'appréciation est élevé. L'agent peut en effet choisir d'adopter un comportement dit opportuniste, c'est-à-dire choisir de " trahir » au moins partiellement les intérêts du principal pour mieux servir les siens propres (en minimisant ses efforts, par exemple). Ce risque est habituellement dénommé "risque moral ". Les exemples de relation d'agence sont nombreux : conseiller/client, assureur/assuré, actionnaires/dirigeant, donneur d'ordres/soustraitant, etc., la plus fameuse restant celle de tout un chacun avec son garagiste. On ne peut observer l'effort et la qualité du travail fourni par son garagiste que de façon imparfaite, et bien souvent au travers de déclarations ou de rapports émanant du garagiste lui-même ; il y a donc risque moral.

Ainsi, les hypothèses de base de la théorie de l'agence sont les suivantes : divergence entre les préférences de l'agent et les objectifs du principal, coût du contrôle des résultats ou des actions de l'agent et risques de comportement opportuniste lié à l'imperfection du contrôle. Face à cela, l'objectif de la théorie de l'agence est de déterminer la modalité de rémunération et, plus généralement, le contrat qui permet de satisfaire le principal et l'agent, et surtout qui réduit la probabilité d'opportunisme de l'agent. La recherche des modalités de contrôle de l'agent (système d'évaluation et de mesure de la performance) et l'établissement de structures incitatives optimales (système d'incitation qui spécifie les sanctions et récompenses associées à la performance mesurée) sont alors l'objet de la théorie de l'agence (1).

Se situant dans cette perspective, la New economics of personnel (branche appliquée de la théorie des incitations) recherche donc les moyens dont dispose le principal pour motiver l'agent dans l'accomplissement de sa mission. Elle insiste particulièrement sur le fait qu'une modification du système de rémunération de l'agent l'incitera à maximiser ses efforts. Dans les modèles de rémunération en fonction des résultats, dont nous présenterons la logique de construction et les principales conclusions, si le rôle de l'agent est toujours tenu par le seul enseignant, le principal peut être représenté, selon les besoins de l'analyse, par l'État (représenté localement par les corps d'inspection), le directeur d'établissement ou les parents d'élèves.

Le principe mis en œuvre paraît d'une grande simplicité (si l'on fait abstraction des difficultés inhérentes au dispositif et au contexte particulier, difficultés sur lesquelles nous aurons l'occasion de revenir par la suite). Le paiement au résultat ou à la performance vient se substituer au paiement à l'effort lorsque l'effort est non observable et que le résultat est une fonction de l'effort. L'effort est celui de l'enseignant, le résultat est le résultat scolaire de l'élève. L'effort de l'enseignant n'est pas connu, parce que non directement observable, mais ses conséquences sont mesurées par le résultat de l'élève (au travers d'un test, par exemple), qui permet de livrer par hypothèse une estimation sans biais de l'effort de l'enseignant. Le niveau de performance scolaire de l'élève est mesuré en acceptant une marge d'erreur mais en supposant que ces erreurs sont nulles en moyenne. La satisfaction globale de l'enseignant est mesurée par la différence entre sa rémunération et le coût généré par son effort. Les résultats du modèle sont les suivants:

- Si la rémunération de l'enseignant est fixe, indépendante de l'effort, alors le niveau réel d'effort sera choisi par l'enseignant en fonction de ses intérêts et de l'équivalence perçue entre son effort et sa rémunération ;

- Si le salaire de l'enseignant comprend une partie fixe et une partie variable, fonction du résultat de l'élève, autrement dit, dans le cas d'un paiement partiellement en fonction du résultat, l'enseignant sélectionnera son niveau d'effort de telle sorte que 
le gain lié au supplément d'effort (le gain correspond à l'augmentation de la rémunération) égalise le coût généré par le supplément d'effort (le coût peut, par exemple, correspondre à l'énergie dépensée ou au nombre d'heures de travail de préparation).

Les enseignements généraux du modèle s'en déduisent facilement. On observe tout d'abord un arbitrage incitation/risque dès lors que l'agent a un comportement d'aversion pour le risque (l'aversion au risque est un comportement économique selon lequel les agents préfèrent un gain relativement sûr à un gain bien plus important mais aléatoire). On en déduit que le salaire moyen de ce type de rémunération doit être plus élevé que celui versé dans le cas d'une rémunération fixe. On voit ensuite que les conditions de la précision de l'estimation ont aussi un impact direct sur la rémunération. Ainsi, une augmentation de la probabilité d'erreur de l'évaluation des résultats scolaires des élèves diminue directement le pouvoir incitatif de la rémunération, de même qu'une augmentation de l'aversion au risque de l'agent (2). Ajoutons enfin que le type de contrat adopté devra tenir compte de la nature du travail et de l'activité de production car le coût d'évaluation de l'effort et/ou des résultats, ainsi que la possibilité pour l'agent d'adopter un comportement opportuniste, varient avec la nature de l'activité de travail ; pour l'activité d'enseignement, il conviendra de prendre en compte notamment la multiplicité des tâches réalisées ainsi que l'incertitude des résultats tenant aux caractéristiques individuelles des élèves, à l'effort et aux attitudes des autres enseignants ainsi qu'à l'environnement de la classe.

Cette présentation, volontairement simplifiée, donne le ton des contributions qui utilisent le cadre et les outils de la théorie de l'agence pour analyser la gestion du personnel enseignant. Cette insertion de l'enseignant dans une relation bilatérale apparaît comme la tentative la plus récente, en économie, de rentrer dans le fonctionnement organisationnel d'une institution qui était restée jusqu'à présent à l'état de " boîte noire ». En proposant ces outils pour ouvrir la « boîte noire ", la théorie de l'agence ouvre en même temps de nouvelles perspectives pour une analyse économique du système éducatif, à travers la construction de nouvelles grilles de lecture des mécanismes organisationnels. En même temps, elle ouvre le champ à une réflexion sur les difficultés d'application pratique des mécanismes incitatifs au secteur de l'enseignement. En effet, au-delà de la simplicité apparente de ce modèle, la mise en œuvre de dispositifs incitatifs dans la gestion du personnel enseignant s'avère complexe pour diverses raisons que nous allons envisager. La première de ces raisons est que les schémas incitatifs reposent sur des résultats qui sont souvent difficilement évaluables empiriquement.

\section{LA MISE EN INCITATION DES ENSEIGNANTS : DES DIFFICULTÉS TECHNIQUES D'ÉVALUATION}

Rémunérer les enseignants pour partie en fonction de leur performance individuelle suppose que l'on sache évaluer le travail des enseignants, c'est-à-dire que l'on dispose d'indicateurs permettant de mesurer leur effort et/ou les résultats de leur effort. Le choix de ces indicateurs constitue un défi majeur car l'évaluation est toujours un enjeu social (Richardson, 1999). Le modèle présenté ci-dessus propose d'utiliser comme indicateur les résultats des élèves aux tests, mais d'autres indicateurs ou méthodes d'évaluation existent. À partir de leur examen, nous verrons que la mise en incitation des enseignants se heurte toujours à la même difficulté : assurer l'intégrité et la crédibilité de l'évaluation.

\section{L'évaluation subjective de la performance}

Elle fait dépendre la rémunération de l'enseignant des évaluations de son ou ses supérieurs hiérarchiques, une bonne évaluation permettant au salarié d'obtenir un salaire plus élevé ou une prime. Ce type d'évaluation a l'avantage (Prendergast, 1999) de surmonter le problème délicat des tâches multiples (cf. infra) car il permet d'évaluer l'enseignant sur un ensemble d'activités et de tâches (enseigner, organiser, communiquer...) qui contribue à la performance ; il prend en compte le travail de l'enseignant dans sa globalité. L'étude récente de Jacob \& Lefgren (2005) concernant un échantillon d'enseignants du primaire d'un district américain, montre que les évaluations subjectives de 202 instituteurs, pratiquées par les directeurs d'écoles, constituent de bien meilleurs prédicteurs des résultats scolaires des élèves que les diplômes ou l'expérience professionnelle des maîtres.

Ce système d'évaluation a aussi les défauts de son caractère subjectif (Tirole, 1992). Le premier est le risque de compression des classements, qui signifie que les superviseurs ont tendance à ne différencier que faiblement les bonnes et les mauvaises performances. De tels comportements ont pour effet de réduire l'importance de l'évaluation subjective dans les dispositifs incitatifs puisque la relation entre 
l'effort et la rémunération est fortement parasitée. Ce risque de compression des classements apparaît dans l'étude de Jacob \& Lefgren (2005), dans laquelle l'évaluation subjective se révèle pertinente pour identifier les meilleurs enseignants ou les plus médiocres, mais ne permet pas de différencier les enseignants moyens entre eux et semble, en outre, porteuse de discriminations à l'encontre des hommes et des enseignants non titulaires. Le second défaut est le risque que les enseignants adoptent des comportements visant à " impressionner » le superviseur et à suggérer qu'ils sont plus efficaces que leurs collègues afin d'augmenter la probabilité d'obtenir une meilleure notation. Au sein des établissements scolaires, ces activités dites d'influence visent par exemple la recherche d'avantages et se manifestent sous la forme de jeux de pouvoir ou d'altération d'informations (Milgrom \& Roberts, 1997). Elles génèrent une double distorsion : d'une part, le temps et l'énergie consacrés à exercer une influence auraient pu être plus efficacement utilisés sur des tâches productives ; d'autre part, il est très difficile de savoir si l'enregistrement d'une bonne performance renvoie à du favoritisme ou à une bonne performance véritable. Le troisième défaut de l'évaluation subjective réside dans la nature difficilement objectivable de l'activité d'enseignement : l'absence d'une relation nette entre les actions d'un enseignant et l'apprentissage de l'élève fait que les superviseurs ne sont pas en mesure d'expliquer pourquoi un enseignant est plus efficace qu'un autre, ni d'indiquer clairement les étapes à suivre et les comportements à adopter pour améliorer la performance (Murname \& Levy, 1996). De ce fait, la confiance des enseignants dans le processus d'évaluation diminue et leur motivation s'affaiblit.

\section{L'évaluation objective de la performance}

Elle base les primes des enseignants soit sur une mesure de leur valeur ajoutée, soit sur les résultats des élèves à des tests (Bacharach et al., 1984 ; Kane \& Staiger, 2002). Cela résout le problème de l'évaluation subjective mais pose d'autres problèmes.

Les tentatives de mesure de la valeur ajoutée des enseignants se sont fortement développées aux États-Unis parallèlement aux progrès techniques importants réalisés en matière de construction de systèmes de données. Encouragés pour certains par l'octroi de subventions du Département de l'éducation, les États et les districts ont rapidement développé des bases de données longitudinales pour le suivi des élèves, permettant une estimation des contributions en valeur ajoutée des districts, des établissements et des enseignants. Les systèmes d'évaluation mis en place et leurs résultats ont ensuite fait l'objet de nombreuses recherches (3). Ces recherches concluent toutes à l'existence de différences importantes et persistantes entre les résultats des élèves en fonction de leur enseignant, suggérant que les enseignants ont des valeurs ajoutées différenciées et donc une influence importante sur les résultats de leurs élèves, influence mesurée sur plusieurs années. Signalons la convergence de ces résultats avec les recherches françaises, notamment celles menées par I'IREDU sur l'« effet-maître » (Mingat, 1991 ; Bressoux, 1994 ; Duru-Bellat, 2001). Ces études, américaines et françaises, restent néanmoins très prudentes quant à l'interprétation de cet effet-maître, soulignant le risque de l'attribuer aux caractéristiques personnelles de l'enseignant, sans considérer les autres variables de contexte, comme par exemple le contexte établissement. Elles s'interrogent également sur la capacité de ces estimations à guider la pratique éducative et à être exploitées comme incitations pour que les enseignants modifient leurs pratiques. En France aujourd'hui, les indicateurs de valeur ajoutée proposés par le ministère de l'Éducation nationale sont le taux de réussite au baccalauréat, le taux d'accès au baccalauréat et la proportion de bacheliers parmi les sortants. Ils mesurent la valeur ajoutée des établissements (et non directement des enseignants) en pondérant les résultats des élèves par des indicateurs sociaux comme, par exemple, l'origine sociale des élèves. N'ayant pas servi de support à la mise en place d'un système de récompenses, ils n'ont, pour I'heure, pas suscité de recherches empiriques.

L'autre type d'évaluation objective consiste à mesurer la performance des enseignants à partir des résultats des élèves à des tests. Le premier problème posé par ce type d'évaluation est que les tests standardisés ne fourniraient pas une très bonne mesure des qualifications des élèves et donc des fruits du travail de l'enseignant. Le second problème est que l'évaluation par les tests incite les enseignants à délaisser les activités non mesurables (Holmström \& Milgrom, 1991). Selon Murnane \& Cohen (1986), même si les tests fournissaient des mesures exactes des compétences des élèves dans des domaines particuliers, le problème des incitations pour allouer le temps stratégiquement à des élèves particuliers et à des domaines particuliers et pour négliger les aspects du métier non mesurables par des tests standardisés demeurerait. On peut également penser qu'à l'approche de l'échéance que constitue le test, 
un enseignant estimant que ces élèves n'ont pas acquis les compétences minimales suffisantes pour réussir le test pourra se sentir découragé et réduira ses efforts. Enfin, de nombreux auteurs mettent en avant le fait que ce système incite les enseignants à enseigner pour le test (teaching to the test) (4). Anne West \& Hazel Pennell (2005) constatent que « cette pratique a des effets dysfonctionnels, comme par exemple la réduction de l'ampleur et de la diversité des programmes ou la priorité accordée aux entraînements aux tests dans le cursus scolaire [...] L'importance donnée à ces derniers a pour effet de focaliser l'attention sur les processus purement internes aux établissements comme moyens privilégiés d'améliorer l'apprentissage et les savoirs, plutôt que sur les problèmes structurels d'hétérogénéité et d'inégalité sociale des élèves. Enfin, les pressions liées à l'utilisation de ces tests sont telles pour les établissements que leur usage continu ne permet pas de mesurer les progrès réels des élèves dans les processus d'apprentissage. »

\section{L'évaluation de la performance relative}

Elle implique que les agents sont partiellement rémunérés en fonction de leurs résultats relativement aux résultats obtenus par leurs collègues. Un dispositif de ce type a été mis en place en Israël en septembre 2001, dans 90 établissements volontaires de l'enseignement secondaire. Selon ce dispositif, rapporté par Victor Lavy (2003), les enseignants peuvent obtenir à titre individuel des primes monétaires en contrepartie d'une amélioration des performances de leurs élèves aux examens. Dans quatre disciplines sélectionnées (hébreux, arabe, mathématiques et anglais), l'enseignant reçoit une gratification sur la base de sa performance relative vis-à-vis des autres collègues de sa discipline. Cette performance relative est mesurée selon deux critères : le taux de passage des élèves et le score moyen de la classe à chaque examen. L'enseignant a la possibilité de « jouer » autant de fois qu'il a de classes différentes. D'après Victor Lavy, le programme s'est révélé effectivement incitatif, notamment en matière de changements de comportement des enseignants (effort accru particulièrement avant les examens, mais aussi changement de méthodes via une écoute et une attention plus soutenue aux besoins des élèves). Alternative crédible (Lavy, 2003) à l'évaluation objective en cas de non-consensus sur les « bons » indicateurs, l'intérêt de ce dispositif se situe dans une plus grande maîtrise budgétaire. Mais le fait que les enseignants ne sont plus jugés sur leur propre performance, mais par rapport à celle des autres, entraîne des risques de démotivation et de dégradation du climat de travail.

Au terme de cette section, il apparaît que l'évaluation de la performance des enseignants, en liaison avec la question de la mise en œuvre de mécanismes incitatifs, offre des réflexions et des résultats de recherche contradictoires. Mais de nombreuses études sur le sujet attribuent le faible impact des incitations aux difficultés d'évaluation qui conduisent à un trop faible écart entre les primes attribuées aux plus productifs et celles attribuées aux moins productifs, enlevant au programme sa fonction motivante (Malen, 1999 ; Pearce \& Perry, 1983). Nous verrons que les difficultés d'évaluation et le faible écart entre les primes ne sont pas les seuls obstacles à la mise en œuvre des incitations monétaires.

\section{LA MISE EN INCITATION DES ENSEIGNANTS : DES OBSTACLES LIÉS AUX CARACTÉRISTIQUES DE L'ACTIVITÉ DE TRAVAIL}

Associés aux difficultés techniques d'évaluation, trois autres obstacles apparaissent à la mise en incitation des enseignants, liés aux caractéristiques de leur activité de travail.

\section{La multiplicité des objectifs et des principaux}

Elle trouve son origine dans le caractère public de l'offre d'éducation qui implique de prendre en compte les différents intérêts des diverses parties prenantes. II s'agit là d'une particularité du secteur public, souvent illustrée par l'idée que l'agent (l'enseignant) doit servir plusieurs « maîtres » - techniquement, il travaille pour plusieurs principaux (Burgess \& Rato, 2003). Développer des incitations dans ce type de circonstances est particulièrement complexe (Bernheim \& Whinston, 1986 ; Dixit, 1996, 1997 ; Holmström \& Milgrom, 1991) parce que les principaux n'ont pas les mêmes objectifs et que leurs intérêts ne sont pas identiques.

Le système éducatif est connu pour avoir de nombreuses parties prenantes dont certaines vont intervenir à titre de principaux externes dans une relation d'agence qui se joue aussi dans l'arène politique. Dans cette optique, Avinash Dixit (2002) en vient à répertorier une liste qui ne comprend pas moins de 
sept groupes d'acteurs majeurs. Celle-ci inclut les parents et les enfants, les enseignants et leurs syndicats, les contribuables au niveau local ou national, les employeurs potentiels des diplômés, la société envisagée globalement, les établissements privés, les groupes favorables ou opposés à certains items spécifiques du programme officiel. Ces groupes ont des préférences variées et diverses sur des objectifs éventuellement antagonistes. Les parents peuvent attendre de l'école qu'elle dispense " une bonne éducation ", qu'elle assure la garde des enfants ou un niveau de formation au-delà du secondaire; les enseignants et leurs syndicats désirent des salaires élevés, de bonnes conditions de travail, impliquant des classes à effectif plus réduit, un accès à des formations leur permettant d'améliorer leur qualification professionnelle ; les contribuables souhaitent des coûts faibles ; les employeurs attendent des qualifications professionnelles pointues mais aussi suffisamment larges pour assurer l'employabilité, la capacité de résolution des problèmes et le développement des "bonnes " attitudes ; la société, envisagée comme un tout, veut de "bons " citoyens ; les établissements privés sont concurrents des établissements publics tant en ce qui concerne les élèves que les fonds publics.

Comme le souligne Avinash Dixit (2002), les différents objectifs des différents principaux ne sont pas nécessairement contradictoires mais, étant donné les ressources limitées des établissements et des enseignants, ils peuvent entrer en concurrence et donc devenir fortement substituables dans le processus d'éducation. Au plan théorique comme au plan empirique, l'existence de plusieurs principaux affaiblit mécaniquement le poids des incitations globales (Laffont \& Tirole, 1993) car chaque principal voudra affecter un coefficient positif à l'élément qui le concerne au premier chef et un coefficient négatif aux autres dimensions. Cela crée une externalité négative (5) pour les autres principaux qui font face à de plus faibles efforts en faveur de leur propre objectif. Quand les efforts des agents pour les différents principaux sont "substituts ", l'agent tendra à allouer son effort au principal qui paie les plus hauts coefficients et cela renforcera les effets des externalités négatives et donc affaiblira les incitations agrégées.

\section{La multiplicité des tâches}

Associé à la pluralité des principaux, le deuxième obstacle à mesurer la performance individuelle des enseignants est lié à la multiplicité des tâches qu'ils effectuent. L'OCDE (2005b) indique quelques-uns des domaines auxquels s'applique cette responsabilité élargie des enseignants. Au niveau individuel : mettre en route et gérer les processus d'apprentissage, répondre efficacement aux besoins individuels des apprenants, intégrer l'évaluation formative et sommative. Au niveau de la classe : enseigner à des classes pluriculturelles, développer de nouvelles aptitudes de manière transversale à tout le programme, intégrer les élèves présentant des besoins éducatifs spécifiques. Au niveau de l'établissement scolaire : travailler en équipe, évaluer, organiser des programmes de développement organisationnel scolaire, utiliser des TIC dans l'enseignement et l'administration. Au niveau des parents et de la collectivité élargie : conseiller les parents de manière professionnelle, mettre en place des partenariats avec les collectivités locales. Les missions de l'enseignant sont donc multiples et particulièrement variées, ce qui complique grandement les mécanismes incitatifs. En effet, dans un contexte multitâche, les agents choisissent d'allouer leur travail aux diverses tâches de façon différenciée (Dewatripont et al., 1999) selon les bénéfices relatifs qu'ils peuvent obtenir sur les diverses tâches mais aussi selon le caractère complémentaire ou substituable des tâches à effectuer. Dans cette logique, la décision de récompenser l'exercice d'un sousensemble d'activités ou de tâches particulières (Holmström \& Milgrom, 1990) risque de susciter une réallocation du travail en faveur de celles qui sont directement récompensées et au détriment de celles qui ne le sont pas.

Une solution à ce problème pourrait être la spécialisation des tâches (Hannaway, 1992) et leur répartition auprès de personnels différenciés. Ce type de solution n'est bien sûr envisageable qu'à partir d'une certaine taille d'école ou d'établissement. La diversité des fonctions exigées des enseignants amène en effet à se demander s'il ne conviendrait pas de cibler davantage leurs compétences spécialisées sur la satisfaction des besoins des élèves et de réaffecter certaines de leurs tâches actuelles autres que l'enseignement à d'autres professionnels ou personnels de soutien. Cette solution apparaît cohérente mais sa faisabilité dépend fondamentalement de la capacité à segmenter les tâches et les résultats de l'éducation (Dixit et al., 2000). En France, par exemple, la présence ou l'intervention d'éducateurs, de médiateurs et de psychologues (remise en cause aujourd'hui) dans certains établissements pourrait accréditer la thèse de la spécialisation. Cependant, l'efficacité de leur travail passe par la concertation et la permanence 
du dialogue avec l'équipe enseignante. II ne s'agit donc pas d'une spécialisation au sens où les uns ne sont pas concernés par les tâches des autres, mais plutôt d'un élargissement et d'un enrichissement de la conception pédagogique qui impliquent de regrouper différents professionnels concernés par le même problème et qui vont agir chacun à leur niveau. Les résultats obtenus par chacun dépendront alors notamment du degré de coopération entre les intervenants et de leur confiance mutuelle (Baker, 1989 ; Itoh, 1991 ; Lazear, 1989 ; Milgrom, 1988). Les programmes personnalisés de réussite éducative (PPRE) en France en constituent une illustration concrète (6). La question de la prise en compte de la multiplicité des tâches rejoint alors celle de la reconnaissance du travail d'équipe.

\section{Le travail d'équipe}

Le troisième obstacle réside dans la difficulté à mesurer la performance individuelle de l'enseignant dans le cadre d'un travail qui implique l'existence d'une équipe, simple support technique et pédagogique ou équipe active favorisant le travail de groupe. Partie immergée de l'activité de l'enseignant, le travail d'équipe en constitue pourtant une composante importante, présente tant au niveau disciplinaire qu'au niveau extra-disciplinaire (7).

En matière d'incitations, les complications inhérentes à la production en équipe sont directement associées à l'asymétrie de l'information. Soit il n'est pas possible de mesurer la contribution de chacun dans la production collective du groupe (Alchian \& Demsetz, 1972) car seul le résultat global est visible ; soit les contributions individuelles sont visibles mais l'effort ou le résultat d'un agent donné dépend des actions des autres agents. En conséquence, il est respectivement, soit impossible, soit délicat, de fonder le système de rémunération sur les contributions individuelles des agents :

- Dans le premier cas, l'imparfaite observabilité des contributions individuelles est propice au comportement de "passager clandestin ", chaque membre de l'équipe réduisant son effort, espérant bénéficier du travail des autres. Ces comportements induisent une réduction du résultat global. On retrouve ici, en effet, le problème classique selon lequel la contribution individuelle et la rémunération se diluent au rythme de la croissance de la taille de l'équipe ;

- Dans le second cas, celui où les efforts individuels sont observables mais où le résultat de chacun dépend des actions des autres membres de l'équipe, des rémunérations en fonction des performances individuelles peuvent inciter les enseignants à négliger le travail d'équipe qui contribue à l'accomplissement des objectifs généraux de l'établissement pour se concentrer sur les objectifs spécifiques. Elles sont susceptibles également d'engendrer une absence de coopération entre enseignants qui peut être coûteuse étant donné d'une part, les éléments de production d'équipe dans l'enseignement et d'autre part, une contribution individuelle appliquée au résultat scolaire global qui ne peut être totalement identifiée. Ainsi, lorsque la coopération entre travailleurs est importante pour les objectifs de l'organisation, des rémunérations partiellement individualisées peuvent réduire la performance de l'équipe (8) en augmentant le coût marginal de l'effort de coopération (Milgrom \& Roberts, 1997). Les mécanismes d'incitations individuelles apparaissent alors potentiellement porteurs de paradoxe. Ils visent à améliorer la performance collective de l'établissement en utilisant les outils d'évaluation de la performance individuelle. Ils surestiment ou sous-estiment probablement cette performance individuelle dont on peut penser qu'elle est assez fortement liée respectivement à la qualité ou au déficit d'organisation et de mobilisation collective.

Ces éléments (prise en compte de la multiplicité des principaux, de la multiplicité des tâches et d'un travail d'équipe) propres à la nature de l'activité de travail des enseignants, témoignent de véritables difficultés à mettre en œuvre efficacement des incitations monétaires visant à augmenter la performance des enseignants et, par ricochet, celle de leurs élèves. Des freins d'ordre culturel peuvent s'y ajouter.

\section{LA MISE EN INCITATION DES ENSEIGNANTS : UN DÉFI CULTUREL}

La question de la mise en incitation du point de vue de la « culture enseignante " sera abordée dans une de ses dimensions fondamentales puisqu'il s'agira ici de tenter de déterminer dans quelle mesure les incitations monétaires rencontrent les motivations des enseignants. À l'origine de cette question, la théorie des attentes (Lawler \& Nadler, 1977 ; Porter \& Lawler, 1968 ; Vroom, 1964) analyse le processus qui conduit à la motivation (pour une analyse actualisée, voir Roussel, 1996 et 2000) ; les travaux américains en science administrative et en management public 
(Alonso \& Lewis, 2001 ; Crewson, 1997 ; Perry, 1996, 1997 ; Perry \& Wise, 1990) tentent d'identifier les motivations spécifiques des travailleurs de la sphère publique, notamment à partir de la distinction entre motivation intrinsèque et extrinsèque (9). Ces travaux conduisent à interroger la capacité des incitations monétaires fondées sur des évaluations individuelles à augmenter la motivation et, partant, le niveau d'effort et de performance des enseignants. Précisons ici que le lien motivation/performance n'est pas déterministe (Levy-Boyer, 2001). D'une part, la performance s'explique aussi par d'autres facteurs comme les compétences et les conditions de travail, d'autre part, la motivation peut conduire à une augmentation de l'effort sans pour autant accroître la performance.

Une façon simple d'aborder la question est de considérer les enquêtes sur les motivations des enseignants (débutants et expérimentés). Concernant les choix effectués en amont, c'est-à-dire au moment de la préparation aux métiers d'enseignant par les étudiants, les études sont peu nombreuses mais elles montrent que l'influence des salaires relatifs apparaît faible, à la fois globalement et par discipline (Hanushek \& Pace, 1995). Pour les enseignants déjà en poste, les résultats sont plus différenciés, nous le verrons.

Une enquête du ministère de l'Éducation nationale (2001) en France, portant sur les enseignants du primaire débutants et expérimentés montre que les facteurs intrinsèques sont prépondérants. Parmi les trois raisons principales invoquées, on trouve le désir d'enseigner (environ $70 \%$ des enseignants dans chacun des deux groupes, débutants et expérimentés, ont cité cette raison parmi les trois principales), le désir de s'occuper d'enfants (environ $60 \%$ ) et le désir d'exercer une fonction éducative (environ $40 \%$ ). Les facteurs extrinsèques apparaissent moins déterminants : la sécurité de l'emploi, le temps libre et le salaire sont cités respectivement par environ $20 \%$, $10 \%$ et $2 \%$ des enseignants de chaque groupe. On trouve des résultats analogues dans beaucoup d'autres pays (OCDE, 2005c) : travailler avec les enfants, s'épanouir intellectuellement et contribuer à la société sont des raisons majeures qui incitent à choisir et à demeurer dans l'enseignement. Ainsi, les études confirment l'existence d'une motivation intrinsèque, même si le caractère déclaratif des réponses doit conduire à une interprétation prudente. D'ailleurs, les facteurs liés à la situation personnelle prennent plus d'importance à partir du moment où l'intéressé enseigne : la compatibilité avec la vie privée, le temps libre, la stabilité de l'emploi et la flexibilité des horaires apparaissent alors comme des aspects importants de satisfaction personnelle.

Au vu de ces résultats, on peut penser que les considérations non pécuniaires jouent un rôle plus fort que dans les autres professions. D'autres indices en sont fournis dans les études sur la mobilité des enseignants, études qui montrent qu'elle dépend plus des caractéristiques des élèves des établissements (caractéristiques ethniques et faiblesse des niveaux scolaires) que des aspects financiers (Bennell, 2004 ; Hanushek et al., 2004 ; Hoxby, 1996). De même, la liberté pédagogique, l'exercice d'une responsabilité, ainsi que d'autres motifs intrinsèques seraient premiers parmi les facteurs de motivation des enseignants américains (Sylvia \& Hutchinson, 1985). La théorie des différences compensatrices (10) semblerait donc s'appliquer au domaine (Baugh \& Stone, 1982). Par exemple, la réduction de la taille de la classe peut, pour Kirby \& Grissmer (1993), être davantage interprétée comme un indice de qualité de vie de l'enseignant que comme une mesure destinée à améliorer les performances des élèves.

De façon générale, les études réalisées sur les spécificités des salariés du public par rapport à leurs homologues du privé vont dans le sens d'un particularisme des agents publics relativement à la rémunération en tant que facteur incitatif. Hal Rainey (1979, 1982) comparant 275 cadres intermédiaires appartenant aux deux secteurs montre que les agents publics présentent une plus forte sensibilité à des desseins altruistes et éthiques et sont moins sensibles que les agents privés aux récompenses monétaires. D'autres études plus récentes témoignent également des préférences différenciées des agents selon leur appartenance au secteur public ou au secteur marchand. Michelle Brown (2001) montre que les employés du secteur public valorisent particulièrement les objectifs altruistes (aider, rendre service), considérés comme une des spécificités de leur activité de travail. Sue Frank \& Gregory Lewis (2004) montrent que la motivation des agents du public serait plus fortement liée à des facteurs intrinsèques (intérêt du travail, utilité sociale), tandis que la motivation des agents du privé serait relativement plus liée à des facteurs extrinsèques (rémunération et carrière). Une étude de I'OCDE (2005a), analysant les conséquences de la mise en place d'une rémunération liée à la performance dans 14 pays conclut que "les perspectives de carrière et de promotion et l'intérêt du travail exercé s'avèrent être les premiers facteurs de motivation pour les fonctionnaires, loin devant l'incitation "rémunération à la performance". " (p. 14) 
La notion de " service public » aurait donc un sens dans la réalisation concrète de l'activité de travail des agents du secteur public et déterminerait (en association avec d'autres facteurs bien sûr) leur degré de motivation, de mobilisation et d'engagement. Les «valeurs » qui animent les fonctionnaires, dont les enseignants, seraient davantage intrinsèques et constitueraient un des déterminants majeurs de leur motivation, questionnant ainsi la place et le rôle des mécanismes incitatifs fondés sur la seule contrepartie monétaire, déterminant mineur (11). L'introduction d'une distinction entre le travail et le rôle complique encore davantage la mise en œuvre de mécanismes incitatifs pour les enseignants. Cette distinction dite " job-role differentiation ", introduite par Ilgen \& Hollenbek (1990), établit que des tâches additionnelles ou émergentes s'ajoutent aux tâches qui définissent objectivement le travail. Ces tâches subjectives émanent de l'environnement social comme du travailleur et spécifient le rôle. Ainsi, le travail est défini comme " un ensemble de tâches élémentaires identifiées sous une dénomination commune et devant être accomplies par un seul individu ", tandis que le rôle renvoie à " un ensemble élargi de tâches, incluant, au-delà des principales caractéristiques du travail, des éléments diffusés et entretenus par le système social ». L'existence d'une réalité subjective dans le travail, d'un rôle, différencié de l'activité de travail objective, concorde assez bien avec la multiplicité des objectifs dévolus aux enseignants via le système éducatif (cf. supra). Mais si ce qui est attendu des enseignants est tout autant un rôle qu'une activité de travail donné, comment alors y associer des incitations monétaires individuelles ?

Plus encore, on peut légitimement se demander si les incitations monétaires pourraient avoir des effets contre-productifs. Comme le soulignait Canice Prendergast (1999), les incitations créent des changements de comportements, mais pas toujours dans le sens attendu (12). Elles peuvent en effet générer un effet d'éviction, diminuant les incitations intrinsèques à s'investir dans l'activité de travail et à rechercher la performance. D'une part, la démotivation peut provenir du fait que l'individu perçoit les incitations monétaires comme un moyen de contrôler son travail, un moyen qui réduit son autonomie et met en cause sa compétence. II peut en résulter une destruction de la confiance entre les deux parties (Bénabou \& Tirole, 2000). II peut en résulter également une réduction de la motivation intrinsèque à réaliser une tâche, particulièrement lorsqu'y est associée l'atteinte d'un niveau de performance donné. Les enseignants n'attendent pas qu'on leur fixe des objectifs et qu'on leur pro- mette des rémunérations supplémentaires pour se sentir responsables de leur travail (Deci \& Koestner, 1999). Ces mécanismes incitatifs, parce qu'ils rentrent en conflit avec ce sens de la responsabilité, avec la dignité de chacun, avec la motivation intrinsèque peuvent faire basculer des enseignants compétents et performants dans la passivité ou la révolte. Ainsi par exemple, le cas de ces enseignants qui, recevant une note inférieure à celle qu'ils estiment mériter, réduisent leur effort et diminuent la quantité de travail effectué (Murname \& Levy, 1996). D'autre part, les incitations monétaires peuvent, en changeant la nature de la motivation sans pour autant en accroître le niveau global, générer des effets adverses (Koretz, 2002). Le premier (nous l'avons évoqué) consiste, pour les enseignants, à "réallouer " leur temps de façon stratégique, en ne s'occupant que des élèves les plus susceptibles de progresser, en n'enseignant que ce sur quoi les élèves sont susceptibles d'être évalués et en les " surentraînant " à cette forme d'évaluation, en négligeant les tâches non évaluées. Le second est la multiplication de cours complémentaires, en vue de préparer les élèves à l'évaluation à partir de laquelle l'enseignant sera luimême jugé éligible à la rémunération supplémentaire. Le troisième effet adverse se traduit par le développement des comportements de fraude. Même si les données sur le sujet restent rares et difficiles à interpréter, les récents développements de méthodes ambitieuses de détection de la fraude des enseignants (Jacob \& Levitt, 2003b) suggèrent que 4 à $5 \%$ des tests de classe dans les écoles élémentaires de Chicago pourraient être affectés par la fraude. Le développement de ce type de comportement est particulièrement à craindre lorsque les enjeux financiers sont importants pour l'établissement et les enseignants.

Ainsi, la mise en incitation des enseignants apparaît comme un défi culturel dans la mesure où les valeurs des enseignants ou leur degré d'acceptabilité sociale des pratiques de rémunération au mérite ou d'incitations monétaires jouent un rôle central dans le succès ou l'échec de ce mode de rémunération.

\section{LA MISE EN INCITATION DES ENSEIGNANTS : UNE EFFICIENCE ÉCONOMIQUE CONTESTABLE}

La mise en incitation est souvent présentée, par ses opposants, comme l'aboutissement d'une logique exclusivement économique, le terme "économique » 
sous-entendant alors individualisme, mise en concurrence, rentabilité et inféodation pécuniaire. Pourtant les arguments économiques ne manquent pas pour montrer que la mise en incitation monétaire des enseignants n'est pas toujours efficace ni efficiente.

\section{Les incitations monétaires peuvent générer un coût supérieur au gain engendré}

Ce premier argument s'appuie sur la distinction utilisée dans la section précédente entre outils de motivation extrinsèque et intrinsèque. Selon Marylène Gagné \& Edward Deci (2005), l'introduction simultanée d'outils extrinsèques et intrinsèques serait contre-productive en raison de l'effet d'éviction déjà évoqué. Le risque est que la motivation extrinsèque se substitue à la motivation intrinsèque, conduisant soit à une «a-motivation », soit à des situations plus coûteuses, dans lesquelles tout effort ou toute tâche supplémentaire ne serait accepté par l'agent qu'en contrepartie d'une rétribution monétaire supplémentaire. Compte tenu du caractère multitâche des enseignants et compte tenu également des ambitions des États en matière d'éducation (cf. supra), l'efficience des incitations monétaires pourrait être compromise par des coûts cumulatifs générés par les mesures de contrôle et d'incitation.

\section{Les incitations monétaires ne sont pas forcément efficaces dans les situations multitâche}

Lorsqu'une activité regroupe plusieurs tâches, une rémunération égalitaire peut être efficiente. Selon Bengt Holmström \& Paul Milgrom (1990), en effet, si le résultat d'une tâche est très faiblement observable, alors le schéma d'incitation pour une tâche concurrente doit avoir moins de force pour éviter une diversion excessive de l'effort à l'encontre de cette tâche et en faveur de la tâche la plus aisément observable. En d'autres termes, les incitations doivent être réparties entre les diverses tâches. Dans un contexte de risque moral, relatif à deux tâches par exemple, si la performance sur la tâche 1 est mesurée de façon plus précise que la performance sur la tâche 2, on pourrait penser que les incitations doivent être nécessairement plus fortes dans la première tâche que dans la seconde, tout simplement parce que l'agent s'y trouve exposé à un risque de faible compensation. Or il n'en est rien (13). Un contrat, prévoyant une rémunération fixe indépendante de la performance, peut se révéler être un contrat incitatif efficient pour une activité regroupant plusieurs tâches. Tel est le cas notamment dans l'exemple à deux tâches, proposé par Bengt Holmström \& Paul Milgrom (1991), dans lequel l'activité mobilise un effort parfaitement substituable dans la fonction de coût et où une des deux tâches est totalement non observable (variance d'erreurs infinie).

Ce principe de « rémunération égalitaire » (Harris \& Holmström, 1982) s'applique directement au débat sur les dispositifs d'incitations monétaires, destinés aux enseignants en vue d'améliorer la qualité de l'enseignement primaire et secondaire. Dans l'activité des enseignants, on peut, nous l'avons évoqué, distinguer des objectifs spécifiques (qui renvoient à la transmission des connaissances et des compétences) et des objectifs généraux (qui renvoient à la citoyenneté, aux valeurs démocratiques, à l'apprentissage de la civilité, à la réalisation personnelle...) S'il peut paraître raisonnable d'attribuer les progrès en matière d'objectif spécifique à un enseignant que l'on pourra identifier (14), il est bien plus difficile en revanche de mesurer la contribution de chaque enseignant aux autres objectifs plus généraux de l'établissement. On sait alors que, s'il est souhaitable que les enseignants se consacrent à des activités diverses et variées, mais qu'il est impossible de déterminer le volume d'effort consacré à chaque activité, une rémunération de tous les efforts sur une base égalitaire n'est pas irrationnelle et peut être efficiente.

\section{Les incitations monétaires ne sont pas le seul moyen de révéler l'information}

En pratique, la relation d'agence qui concerne l'enseignant s'étale au cours d'une période de temps suffisamment longue pour que l'agent répète un certain nombre de fois ses actions, le principal ayant alors l'opportunité de pouvoir observer plusieurs fois les actions et les contextes dans lesquels elles sont menées. L'intuition est que (Bolton \& Dewatripont, 2005 ; Dixit, 2002 ; Prendergast, 1999), lorsque des relations contractuelles se répètent, il ne convient pas nécessairement de fournir des incitations explicites pour induire un effort durant les premières étapes de la vie professionnelle. Un projet d'incitations indirectes, sous la forme de perspectives de meilleures rémunérations futures, peut s'avérer dans ce cas suffisant. L'idée à la base est que le principal n'a pas, au départ, de grandes certitudes sur l'aptitude de l'agent quand les résultats de celui-ci dépendent de son effort, d'un contexte singulier et de son aptitude. Des interactions répétées permettent alors 
au principal, au fur et à mesure que le temps passe, de mieux connaître l'aptitude de l'agent (15). Les travailleurs au départ de la carrière exercent des efforts (sans contrats explicites) pour influencer les perceptions de leurs aptitudes. Ces efforts vont ensuite graduellement décliner au fur à mesure que l'information est révélée avec, pour corollaire immédiat, que les travailleurs plus anciens auront tendance à vivre sur leur réputation. C'est à ce moment que le besoin de fortes incitations explicites se fait le plus sentir, pour que cette catégorie de travailleurs mobilise leur effort.

De façon générale, les enseignants ont de longues carrières au cours desquelles leurs qualifications peuvent être développées et leurs compétences révélées. De ce fait, les suivis de carrière peuvent s'avérer plus efficaces que des incitations articulées sur des performances de court terme, même dans l'hypothèse où ces dernières pourraient être mesurées avec une précision suffisante. L'argument ne peut cependant pas être invoqué en faveur d'une rémunération calée uniquement sur l'ancienneté.

\section{Les incitations monétaires saisissent difficilement les aspects inter-temporels}

Une autre façon de prendre en compte les aspects inter-temporels consiste à distinguer les efforts faits par les enseignants pour améliorer les résultats de court terme, des efforts visant à améliorer les résultats de long terme. Dans cette perspective, Paul Glewwe et al. (2003) développent le modèle de Bengt Holmström \& Paul Milgrom (1991) en le prolongeant dans une application à l'éducation. Dans leur modèle, l'agent enseignant est supposé, par hypothèse, fournir deux types d'effort : un effort pour promouvoir la connaissance et le savoir et un effort tourné vers la certification ou le signal qui vise à améliorer les résultats dans le court terme (résultat aux tests, par exemple, ou obtention d'un diplôme) mais qui, en revanche, a peu d'incidences sur la connaissance à long terme. L'enseignant peut choisir de pondérer différemment les deux types d'effort. Les auteurs ont bien conscience qu'il existe un continuum de situations entre exercer un effort visant à promouvoir la connaissance à long terme et un effort visant, par tous les moyens, à obtenir de bons résultats aux tests scolaires. Mais cette version bipolaire est néanmoins retenue pour des questions de modélisation. Dans le modèle, le principal observe seulement les résultats aux tests qui dépendent à la fois des connaissances produites par l'effort de l'enseignant au cours du temps et de l'effort actuel mobilisé sur l'aspect « signal ».

Si la rémunération est fixe et indépendante de l'effort, l'enseignant choisira un effort relatif à chaque composante de son activité, en fonction de ses intérêts, de l'importance qu'il attribue personnellement à chaque tâche et de sa propre perception de l'équivalence effort/rémunération. Si l'on change les règles du jeu et que l'on paye en fonction des résultats des élèves au test, les enseignants vont alors réaffecter leurs efforts. Deux situations sont alors envisageables. Si la connaissance à long terme est complémentaire de la connaissance pour réussir aux tests, alors l'enseignant augmentera les deux types d'efforts. Si ce n'est pas le cas, alors l'enseignant augmentera son effort pour un résultat de court terme, au détriment de son effort pour la connaissance de long terme.

Le test empirique du modèle effectué par les auteurs semble corroborer l'hypothèse selon laquelle la croissance des résultats aux tests se fait au détriment d'une véritable accumulation de capital humain. Il en est de même de l'étude de Randall Ebberts et al. (2002), qui compare les résultats de deux high school d'un même comté du Michigan ; l'une est dotée d'un dispositif de rémunération calée sur la rétention des élèves et l'autre fonctionne de manière classique. Les auteurs montrent que si le dispositif incitatif augmente bien la rétention (objectif affiché), il n'a pas d'effet sur les résultats scolaires mesurés par l'indicateur classique (grade point average) ; en outre, il réduit les durées moyennes de présence journalière des élèves et augmente la proportion d'élèves en échec scolaire.

Ainsi, au plan économique, la mise en incitation monétaire des enseignants semble se heurter à des obstacles qui limitent son efficacité et son efficience, particulièrement lorsqu'est adoptée une perspective dynamique des carrières et des connaissances.

\section{CONCLUSION}

Au plan théorique, la théorie de l'agence et la New economics of personnel ont le mérite de poser une question largement ignorée précédemment par l'économie de l'éducation, à savoir la question du système de rémunération des enseignants, envisagée en tant que facteur de motivation et d'encouragement à l'effort (Larré \& Plassard, 2006) et en tant que facteur 
d'augmentation du niveau éducatif d'une nation. Auparavant la liaison n'était, en effet, pas perçue de façon évidente et l'on pouvait même parler, sans trop d'exagération, d'une neutralité parfaite de la rémunération sur le niveau d'effort et de performance. Prenant le contre-pied de cette neutralité, la théorie de l'agence met en exergue l'importance des incitations monétaires, en faisant le pari que l'enseignant ne se démarque pas du comportement de la masse des autres travailleurs au regard des considérations monétaires. Les réformes ou projets de réforme associés à cette conception sont variés : évaluation plus systématique de la performance, hausse sélective de la rémunération, fixation d'objectifs à atteindre, renforcement des standards, mise en place de tests, etc.

D'un point de vue empirique, les analyses soulignent les difficultés d'évaluation liées notamment aux spécificités d'un métier complexe comportant de multiples tâches et une dimension d'équipe, pour lesquelles certains résultats ne peuvent pas toujours être mesurés aisément. Elles montrent aussi la difficile cohérence entre des incitations extrinsèques et des motivations intrinsèques, qui viennent limiter l'usage de systèmes explicites très incitatifs car ces derniers peuvent être à l'origine de dysfonctionnements de comportement. Et l'appareil économique montre le caractère non nécessairement efficace ou efficient des mécanismes incitatifs dans certaines situations.

Si les enseignants ont aujourd'hui besoin que leur travail soit mieux reconnu et récompensé, leur mise en incitation monétaire individualisée, basée sur une vision mécaniste (nourrie d'indicateurs et d'objectifs) de la relation entre rémunération, effort et performance, n'apparaît donc pas comme la voie la plus pertinente. Parce qu'il est difficile d'identifier les nombreuses et diverses tâches que les enseignants sont supposés accomplir avec succès, parce qu'il est difficile de spécifier des objectifs de travail clairs, mesurables, réalistes, motivants, et couvrant l'ensemble des tâches, la mise en incitation des enseignants rencontre plus d'obstacles qu'elle n'offre de perspectives et risque d'exacerber les problèmes de fonctionnement des établissements. Pourtant, le fait de l'envisager est révélateur d'un changement profond de logique dans la gestion du personnel enseignant, puisqu'il s'agit d'indexer en partie la rémunération de l'enseignant sur ce qu'il fait (individuellement ou collectivement) et non plus seulement (ou essentiellement) sur ce qu'il est, en termes d'appartenance statutaire à un corps. Indépendamment de la ques- tion de sa légitimité, ce changement peut prendre d'autres voies que la mise en incitation monétaire individualisée.

En effet, face aux difficultés concrètes de mise en application, d'autres solutions sont envisagées tels les dispositifs d'incitations non monétaires basés sur la carrière, le régime de retraite, la souplesse des temps de travail, le renforcement et la reconnaissance de la formation tout au long de la vie, la reconnaissance des compétences mises en œuvre dans l'activité de travail, etc., auxquels il faut ajouter l'idée de spécialisation des tâches développée par les auteurs du job design. Ces thèmes devront faire l'objet de développements plus explicites dans le champ de l'éducation.

Les dispositifs de type collectif constituent une autre alternative, faisant dépendre une partie de la rémunération des enseignants des performances de l'établissement. Tel est le cas, le plus souvent (mais selon des degrés parfois très variables selon les dispositifs concrets), des dispositifs dits d'« accountability " qui combinent évaluation et incitation, en mettant la première au service de la seconde. L'évaluation est résolument externe et concerne l'établissement. L'idée centrale est que, en mesurant et en communicant les résultats scolaires des élèves, notamment en référence à des standards agréés, les acteurs vont chercher à améliorer leur performance en développant leurs compétences. Le système est envisagé comme source inhérente d'incitation pour pousser les établissements vers les résultats attendus. II est supposé induire des changements de comportement de la part des élèves, des enseignants et des établissements qui vont s'aligner sur les objectifs de performance du système. Mais une autre partie des améliorations attendues provient directement de la création d'un système d'incitations explicite susceptible de conduire à l'innovation et à l'efficience. II est clair que ce déplacement d'une logique totalement liée à la performance immédiate et individuelle, à une inscription des modes de rémunération dans une perspective dynamique et collective évite certaines des critiques portées au système de rémunération individualisée. II n'est cependant pas sans faille car il peut se heurter au problème du " passager clandestin " ou du surinvestissement dans les disciplines objets des tests.

Françoise Larré francoise.larre@toulouse.iufm.fr IUFM de Midi-Pyrénées 
(1) Pour une présentation plus complète du modèle d'agence, voir Kreps (1990), Milgrom \& Roberts (1997), Laffont \& Martimort (2002).

(2) On pourrait montrer également que le paiement en fonction des résultats a des effets au plan de l'auto-sélection (Lazear, 1998) au sens où ce type de rémunération peut attirer les meilleurs et décourager les moins bons. Un tel effet se situe toutefois essentiellement dans la perspective de sélection adverse qui n'est pas envisagée ici.

(3) Les premières recherches ont analysé le Tennessee's value added and assessement system (Wright et al., 1997 ; Ballou et al., 2004). D'autres recherches similaires ont ensuite été menées au Texas (Rivkin et al., 2005), au sein de districts comme le New York City public schools (Kane et al., 2005 ; Boyd et al., 2006) et le Chicago public schools (Aaronson et al., 2003).

(4) Selon l'OCDE (2005a), parmi les établissements scolaires anglais et gallois figurant en bonne place dans les classements, « $57 \%$ ont déclaré avoir augmenté le nombre d'heures d'enseignement théorique des disciplines objets des tests, $41 \%$ avoir consacré davantage de ressources aux aptitudes pédagogiques relatives aux disciplines objets des tests, et $30 \%$ avoir engagé plus de ressources dans les disciplines telles que les mathématiques, couvertes par les tests, au détriment d'autres disciplines." (p. 204).

(5) L'externalité ou effet externe désigne une situation économique dans laquelle l'acte de consommation ou de production d'un agent influe sur la situation d'un autre agent non directement impliqué dans l'action. L'externalité est dite positive quand elle génère un bénéfice externe ; elle est dite négative quand elle génère un coût externe. L'exemple le plus célèbre d'externalité est celui de l'apiculteur et de l'arboriculteur développé par James Meade (1952).

(6) Le PPRE vise l'analyse de la pertinence des aides apportées et leur mise en cohérence. Parfois, en effet, les aides existent mais sont juxtaposées, non concertées, mal articulées de sorte que les intervenants ignorent le contenu et les modalités de leurs actions respectives. Le travail effectué dans le cadre du PPRE porte alors sur l'analyse de l'efficacité et la remise en cohérence.

(7) En matière d'enseignement disciplinaire, la situation d'équipe intervient au sens où la performance de l'élève dans une discipline peut dépendre aussi de son apprentissage dans d'autres disciplines, enseignées par les autres enseignants. Ceci est vrai par niveau mais également sur plusieurs niveaux (Ebberts et al., 2002), comme le montre, dans le premier degré, l'existence d'équipes pédagogiques d'enseignants organisées à la fois sur les niveaux élémentaire et moyen. Cette organisation peut s'appuyer sur les avantages comparatifs des enseignants de sorte que chaque équipe sera responsable des thèmes pour lesquels elle peut apporter la plus grande valeur ajoutée. Au niveau secondaire, les établissements peuvent aussi être considérés comme des équipes ; ceci apparaît clairement lorsque les élèves des établissements sont soumis à des tests standardisés qui conduiront à une évaluation de l'établissement dans son ensemble. La situation d'équipe intervient également au-delà de l'aspect disciplinaire puisque les enseignants ont aussi la charge de l'apprentissage de la civilité ou de la lutte contre l'incivilité, de l'éducation à la vie collective et à la citoyenneté, etc. Richard Murnane \& David
Cohen (1986) le soulignent en disant que pour éliminer la drogue et la violence d'une école, il faut que les enseignants ouvrent la porte de leur classe et travaillent en équipe pour contrôler les actions des élèves en dehors de la classe.

(8) L'enquête de David Marsden (1988) qui examine un système de rémunération au mérite dans le service public britannique de l'emploi et celui des impôts, des hôpitaux publics et des établissements d'enseignement supérieur, conclut que l'impact le plus négatif du dispositif est de dégrader les relations de travail car il entraîne des jalousies et défavorise le travail d'équipe.

(9) Selon Teresa Amabile (1993), les individus sont « intrinsèquement motivés lorsqu'ils effectuent une activité pour le plaisir, l'intérêt ou encore la satisfaction de leur curiosité "; ils sont extrinsèquement motivés quand « ils s'engagent dans une activité pour satisfaire un objectif en dehors de l'activité ellemême ".

(10) La théorie des différences compensatrices, à la suite d'Adam Smith, suggère que la concurrence (parfaite) sur les divers marchés du travail doit conduire à des disparités des salaires résultant uniquement des différences dans la qualité des emplois et dans la compétence des offreurs de travail. Ainsi, des travailleurs de même niveau de compétence percevront des salaires différents si leurs conditions de travail sont différentes. À compétence donnée, la pénibilité d'un travail sera donc compensée par un salaire plus élevé. Pour une discussion complète des obstacles à l'application du principe et un état des lieux sur les travaux empiriques, voir notamment Cahuc \& Zylberberg (2001), chapitre 3.

(11) II ne s'agit pas ici de dire que les enseignants relèvent d'une essence particulière d'humanité qui les rendraient indifférents aux gratifications monétaires, mais de souligner que, pour beaucoup d'entre eux (mais pas tous), elles n'apparaissent pas premières dans leur motivation.

(12) De ce point de vue, la théorie de la justice organisationnelle montre que les agents " évaluent " les outils d'évaluation (Greenberg, 1990) et que le sentiment d'équité ou d'iniquité qu'ils ont par rapport à ces outils a une influence sur leur motivation.

(13) Considérons $c\left(e_{1}, e_{2}\right)$, le coût de l'effort exprimé comme une fonction de l'effort total combinant $e_{1}$, l'effort relatif à la tâche 1 et $e_{2}$, l'effort relatif à la tâche $2, e_{1}$ et $e_{2}$ étant substituts. $S i$ $c\left(e_{1}, e_{2}\right)=c\left(e_{1}+e_{2}\right)$, le pouvoir incitatif sur les deux tâches ne peut diverger sans que l'agent néglige complètement la tâche la moins rémunérée. Ceci conduit à faire usage de systèmes d'incitations plus faibles, même pour la tâche la plus facilement mesurable, dès lors que le principal ne désire pas que l'autre tâche soit totalement négligée.

(14) Aussi raisonnable qu'elle paraisse, cette idée n'en est pas pour autant facilement réalisable quand on connaît la complémentarité des disciplines.

(15) La formalisation initiale, combinant sélection adverse et risque moral, par Milton Harris \& Bengt Holmström (1982), a montré sa robustesse dans les développements et les généralisations ultérieures (Dewatripont et al., 1999 ; Harris \& Holmström, 1982).

\section{BIBLIOGRAPHIE}

AARONSON D., BARRO L. \& SANDER W. (2003). Teachers and student achievement in Chicago public high schools. Chicago [II.] : Federal research bank of Chicago.

ALCHIAN A. \& DEMSETZ H. (1972). «Production. information costs and economic organization ». American economic review, vol. $62, \mathrm{n}^{\circ} 5$, p. 777-795.
ALONSO P. \& LEWIS G. B. (2001). « Public service motivation and job performance: evidence from the federal sector ". American review of public administration, vol. $31, n^{\circ} 4$, p. 363.

AMABILE T. M. (1993). «Motivational synergy: toward new conceptualizations of intrinsic and extrinsic motivation 
in the workplace ». Human resource management review, vol. 3, $\mathrm{n}^{\circ}$ 3, p. 195-201.

BACHARACH S. B., LIPSKY D. \& SHEDD J. (1984). Paying for better teaching merit pay and its alternatives. San Fransisco : Jossey Bass.

BAKER G. (1989). Piece rate contracts and performance measurement error. Graduate school of business, Harvard University.

BAKER G., GIBBONS R. \& MURPHY K. (1994). "Subjective performance measure in optimal incentive contract ". Quaterly journal of economics, vol. 109, $\mathrm{n}^{\circ} 4$, p. 11251156.

BALLOU D., SANDERS W. \& WRIGHT P. (2004). « Controlling for student background in value-added assessment of teachers ". Journal of educational and behavioral statistics, vol. 29, $n^{\circ} 1$, p. 37-66.

BAUGH W. H. \& STONE J. A. (1982). «Mobility and wage equilibration in the educator labor market ». Economics of education review, vol. 2, n 3, p. 253-274.

BÉNABOU R. \& TIROLE J. (2000). "Self confidence and social interactions". Cambridge [Mass.] : NBER working paper $n^{\circ}$ W7585. Disponible sur Internet à l'adresse : <http://papers.ssrn.com/sol3/papers.cfm?abstract_ id=218750> (consulté le 12 juin 2009)

BENNELL P. (2004). "Teacher motivation and incentives in sub-saharan Africa and Asia ». Knowledge and skills for development, Brighton. Disponible sur Internet à l'adresse : <http://www.eldis.org/fulltext/dfidtea.pdf> (consulté le 12 juin 2009)

BERNHEIM B. D. \& WHINSTON M. D. (1986). "Common agency ». Econometrica, vol. 54, n 4, p. 923-942.

BOLTON P. \& DEWATRIPONT M. (2005). Contract theory. Cambridge : MIT Press.

BOYD D., GROSSMAN P., LANKFORD H. \& LOEB S. (2006). "How changes in entry requirements alter the teacher workforce and affect student achievement ». Education finance and policy, vol. 1, $\mathrm{n}^{\circ} 2, \mathrm{p} .176-216$.

BRESSOUX P. (1994). «Note de synthèse : Les recherches sur les effets-école et les effets maîtres ». Revue française de pédagogie, $\mathrm{n}^{\circ} 108$, p. 91-137.

BROWN M. (2001). «Merit pay preferences among public sector employees ». Human resource management journal, vol. 11, $\mathrm{n}^{\circ} 4$, p. 38-54.

BURGESS S. \& RATO M. (2003). "The Role of incentive in the public sector: issues and evidence ". Oxford review of economic policy, vol. 19, $\mathrm{n}^{\circ} 2$, p. 285-300.

CAHUC P. \& ZYLBERBERG A. (2001). Le marché du travail. Paris : De Boeck.

CHARREAUX G. (1999). " La théorie positive de l'agence, lecture et relecture ». In G. Koenig, De nouvelles théories pour gérer l'entreprise du XXI siècle. Paris : Économica, p. 61-141.

CLOTFELTER C., LADD H., VIGDOR J. \& DIAZ R. (2004). « Do school accountability systems make it more difficult for low-performing schools to attract and retain high-quality teachers? ". Journal of policy analysis \& management, vol. $23, \mathrm{n}^{\circ} 2$, p. 251-271.

CRAMPES C. \& LAFFONT J.-J. (1995). "Transfers and incentives in the spanish electricity sector ». Revista española de economía, p. 117-140.
CREWSON P. E. (1997). «Public-service motivation: Building empirical evidence of incidence and effect ». Journal of public administration research and theory, vol. 7, $n^{\circ} 4$, p. 499-518.

DARCOS X. (2006). « Rapport à Monsieur Nicolas Sarkozy et propositions sur la situation morale et matérielle des professeurs en France ". La lettre de l'éducation, n 552.

DECI E. L. \& KOESTNER R. (1999). «A meta-analytic review of experiments examining the effects of extrinsic rewards on intrinsic motivation ". Psychological Bulletin, vol. $125, \mathrm{n}^{\circ} 6$, p. 627.

DEE T. S. \& KEYS B. J. (2004). « Does merit pay reward good teachers? Evidence from a randomized experiment ". Journal of policy analysis \& management, vol. $23, n^{\circ} 3$, p. 471-488.

DEWATRIPONT M., JEWITT I. \& TIROLE J. (1999). «The Economics of career concerns ". Review of economic studies, vol. 66, $\mathrm{n}^{\circ} 1$.

DIXIT A. (1996). The Making of economic policy: a transaction cost politics perspective. Cambridge : MIT Press.

DIXIT A. (1997). "Power of incentives in private versus public organizations ". American economic review, vol. $87, n^{\circ} 2$, p. 378-383.

DIXIT A. (2002). "Incentives and organizations in the public sector: an interpretative review ». Journal of human resources, vol. $37, \mathrm{n}^{\circ} 4$, p. 696-727.

DIXIT A., GROSSMAN G. M. \& GUL F. (2000). « The Dynamics of political compromise ". Journal of political economy, vol. 108, n³, p. 531-568.

DURU-BELLAT M. (2001). « Effets maîtres, effets établissements : quelle responsabilité pour l'école ? ". Revue suisse des sciences de l'éducation, vol. $23, n^{\circ} 2$, p. 321 337.

EBBERTS R., HOLLENBECK K. \& STONE J. (2002). "Teacher performance incentives and student outcomes ". Journal of human resources, vol. $37, \mathrm{n}^{\circ} 4$, p. 913-927.

FRANCE : ministère de l'Éducation nationale (2001). « Devenir professeur des écoles ". Note d'information 01-46.

FRANK S. A. \& LEWIS G. B. (2004). « Government employees: working hard or hardly working? ". American review of public administration, vol. 34, $\mathrm{n}^{\circ} 1$, p. 36-51.

GAGNÉ M. \& DECI E. L. (2005). « Self-determination theory and work motivation ". Journal of organizational behavior, vol. 26, n 4, p. 331-362.

GARY-BOBO R. \& MAHJOUB M. B. (2006). " Estimation of class-size effects, using "maimonides' rule": the case of French junior high schools ". Londres : CEPR $n^{\circ} 5754$.

GLEWWE P., ILIAS N. \& KREMER M. (2003). « Teacher Incentives ». Cambridge [Mass.] : NBER working paper $n^{\circ} 9671$.

GREENBERG J. (1990). «Organizational justice: yesterday, today, and tomorrow ". Journal of management, vol. 16, $\mathrm{n}^{\circ} 2$, p. 399.

HANNAWAY J. (1992). « Higher order skills, job design: an analysis and proposal ». American educational research journal, vol. 29, $\mathrm{n}^{\circ} 1$, p. 3-21.

HANUSHEK E. A. (2003). "The Failure to imput-based schooling policies ». Economic journal, vol. 113, n 485, p. F64-F98. 
HANUSHEK E. A. \& PACE R. R. (1995). «Who chooses to teach (and why)? ». Economics of education review, vol. $14, n^{\circ} 2$, p. 101-117.

HANUSHEK E. A., KAIN J. F. \& RIVKIN S. G. (2004). «Why public schools lose teachers ». Journal of human resources, vol. 39, $\mathrm{n}^{\circ}$ 2, p. 326-354.

HARRIS M. \& HOLMSTRÖM B. (1982). «A theory of wage dynamics ». Review of economic studies, vol. 49, $n^{\circ} 3$, p. 315-333.

HOLMSTRÖM B. (1982). "Moral hazard in teams ». Bell journal of economics, $n^{\circ} 13$, p. 324-340.

HOLMSTRÖM B. \& MILGROM P. (1990). «Regulating trade among agents ". Journal of institutional and theoritical economics, vol. 146, $\mathrm{n}^{\circ} 1$, p. 85-105.

HOLMSTRÖM B. \& MILGROM P. (1991). « Multitask principal agent analysis: incentives contracts, asset ownership and job design ". Journal of law, economics and organization, $\mathrm{n}^{\circ}$ 7, p. 24-52.

HOXBY C. M. (1996). "Are efficiency and equity in school finance substitutes or complements? ". Journal of economic perspectives, vol. $10, \mathrm{n}^{\circ} 4$, p. 51-72.

ILGEN D. \& HOLLENBEK J. (1990). « The structure of work: job design and roles ". In M. Diunnette \& L. Hough, Handbook of industrial and organizational psychology. Palo alto : Consulting psychologists press.

ITOH H. (1991). " Incentives to help in multi-agent situations ». Econometrica, vol. 59, n³, p. 611.

JACOB B. \& LEFGREN L. (2005). «Principal as agents: subjective performance measurement in education ». Cambridge [Mass.] : NBER working paper $n^{\circ}$ W11463. Disponible sur Internet à l'adresse : http://ssrn.com/ abstract=755699 (consulté le 3 juin 2009).

JACOB B. \& LEVITT S. D. (2003a). «Rotten apples: an investigation of the prevalence and predictors of teacher cheating ». Quarterly journal of economics, vol. 118, $\mathrm{n}^{\circ} 3$, p. 843-877.

JACOB B. \& LEVITT S. D. (2003b). " Catching cheating teachers: the results of an unusual experiment in implementing theory ». Cambridge [Mass.] : NBER working paper $\mathrm{n}^{\circ} 9414$.

JENSEN M. C. \& MECKLING W. H. (1976). « Theory of the firm: Managerial behaviour, agency costs and ownership structure ». Journal of financial economics, vol. 3 , $\mathrm{n}^{\circ} 4$, p. 305-360.

KANE T. J. \& STAIGER D. O. (2002). «The Promise and pitfals of using imprecise school accountability measures ». Journal of economic perspectives, vol. 16, $n^{\circ} 4$, p. 91-114.

KANE T. J., ROCKOFF J. E. \& STAIGER D. O. (2005). «Identifying effective teachers in New York City ». Cambridge [Mass.] : NBER summer institute.

KIRBY S. N. \& GRISSMER D. W. (1993). « Teacher attrition: theory, evidence and suggested policy options (P7827) ». Santa Monica [Cal.] : Rand corporation.

KORETZ D. M. (2002). "Limitations in the use of achievement tests as measures of educators' productivity ». The Journal of human resources, vol. $37, \mathrm{n}^{\circ} 4, \mathrm{p} .752$.

KREPS D. M. (1990). «Corporate culture and economic theory ». In J. K. Alt \& K. A. Shepsle, Perspective on positive political economy. New-York : Cambridge university Press, p. 90-143.
LAFFONT J.-J. \& MARTIMORT D. (2002). The Theory of incentives. The principal agent model. Princeton : Princeton University Press.

LAFFONT J.-J. \& TIROLE J. (1993). A Theory of incentive in regulation and procurement. Cambridge : MIT Press.

LARRÉ F. \& PLASSARD J.-M. (2006). « L'enseignant dans tous ses états (économiques) ". Travail et emploi, $\mathrm{n}^{\circ}$ 106, p. 105.

LAVY V. (2002). " Evaluating the effect of teachers' group performance incentives on pupil achievement ". The Journal of political economy, vol. $110, \mathrm{n}^{\circ} 6, \mathrm{p} .1286-$ 1317.

LAVY V. (2003). " Paying for performance: the effect of teachers' financial incentives on students' scholastic outcomes ». Londres : CEPR, $\mathrm{n}^{\circ} 3862$.

LAVY V. (2004). "Performance pay and teachers' effort, productivity, and grading ethics ». Cambridge [Mass.] : NBER working paper $n^{\circ} 10622$.

LAWLER E. E. \& NADLER D. A. (1977). « Motivation: A diagnostic approach ". In R. Hackman, E. Lawler \& L. Porter, Perspectives on behavior in organizations. NewYork : Mc Graw-Hill.

LAZEAR E. P. (1986). «Salaries and piece rates ». Journal of business, vol. 59, p. 405-431.

LAZEAR E. P. (1989). «Pay equality and industrial politics ». Journal of political economy, vol. 97, p. 561-580.

LAZEAR E. P. (1998). Personnel economics for managers. New York : John Wiley \& Sons.

LAZEAR E. P. \& ROSEN S. (1981). « Rank-order tournaments as optimum labor contracts ". The Journal of political economy, vol. 89, $n^{\circ} 5$, p. 841-864.

LÉVY-LEBOYER C. (2001). La Motivation dans l'entreprise. Modèles et stratégies. Paris : Éditions d'organisation.

MALEN B. (1999). « On rewards, punishments and possibilities: Teacher compensation as an instrument for education reform ". Journal of personnel evaluation in education, vol. $12, n^{\circ} 4$, p. 387-394.

MARSDEN D. (1988). «What a performance: Performancerelated pay in the public services ". Londres: Center for economics performance, LSE.

MEADE J. (1952). «External economies and diseconomies in a competitive situation ". The economic journal, vol. $62, n^{\circ} 245$ p. 54-67.

MEURET D. (2001). « Les recherches sur la réduction de la taille des classes ». Paris: Haut conseil de l'évaluation de l'école.

MILGROM P. (1988). "Employment contracts, influence activities, and efficient organization design ». Journal of political economy, vol. 96, $\mathrm{n}^{\circ} 1$, p. 42.

MILGROM P. \& ROBERTS J. (1997). Économie, organisation et management. Bruxelles: De Boeck.

MINGAT A. (1991). «Expliquer la variété des acquisitions au $\mathrm{CP}$ : les rôles de l'enfant, la famille et l'école ". Revue française de pédagogie, $\mathrm{n}^{\circ}$ 95, p. 47-63.

MINGAT A. \& MORLAIX S. (2001). «Améliorer l'efficacité du système éducatif : réflexion sur la complémentarité entre organisation publique et mécanismes de marché ". Administration et éducation, $n^{\circ}$ 90, p. 63-78.

MURNAME R. J. \& LEVY F. (1996). Teaching the new basic skills. New-York: The Free Press. 
MURNANE R. \& COHEN D. K. (1986). « Merit pay and the evaluation problem: why most merit pay plans fail and a few survive ". Harvard educational review, vol. 56, $\mathrm{n}^{\circ} 1$, p. 1-17.

OCDE (2005a). La rémunération liée aux performances dans l'administration. Paris : Éd. OCDE.

OCDE (2005b). Regards sur l'éducation 2005. Les indicateurs de l'OCDE. Paris : Éd. OCDE.

OCDE (2005c). Le rôle crucial des enseignants : attirer, former et retenir des enseignants de qualité. Politiques d'éducation et de formation. Paris : Éd. OCDE.

PEARCE J. L. \& PERRY J. L. (1983). “Federal merit pay: A longitudinal analysis ". Public administration review, vol. $43, n^{\circ} 4$, p. 315 .

PERRY J. L. (1996). " Measuring public service motivation: An assessment of construct reliability and validity ". Journal of public administration research \& theory, vol. $6, \mathrm{n}^{\circ} 1$, p. 5 .

PERRY J. L. (1997). "Antecedents of public service motivation ". Journal of public administration research \& theory, vol. $7, \mathrm{n}^{\circ} 2, \mathrm{p} .181$.

PERRY J. L. \& WISE L. R. (1990). «The Motivational bases of public service ». Public administration review, vol. 50, $n^{\circ} 3$, p. 367-373.

PIKETTY T. (2004). "L'impact de la taille des classes et de la ségrégation sociale sur la réussite scolaire dans les écoles françaises : une estimation à partir du panel primaire 1997 ». Les Dossiers du ministère de l'Éducation nationale. Disponible sur le site : <http://media. education.gouv.fr/file/48/4/2484.pdf> (consulté le 3 juin 2009).

PORTER L. W. \& LAWLER E. E. (1968). Managerial attitudes and performance. Homewood [II.] : Irwin.

PRENDERGAST C. (1999). «The Provision of incentives in firms ». Journal of economic literature, vol. $37, \mathrm{n}^{\circ} 1$, p. 7-63.
RAINEY H. G. (1979). «Perceptions of incentives in business and government: Implications for civil service reform ». Public administration review, vol. 39, $\mathrm{n}^{\circ}$ 5, p. 440-448.

RAINEY H. G. (1982). «Reward preferences among public and private managers: In search of the service ethic ". American review of public administration, vol. 16 , p. 288-302.

RICHARDSON R. (1999). "Performance related pay in schools ". National union of teachers, London school of economics and political science.

RIVKIN S., HANUSHEK E. A. \& KAIN J. F. (2005). « Teachers, schools, and academic achievement ». Econometrica, vol. 73, $n^{\circ} 2$, p. 417-458.

ROUSSEL P. (1996). Rémunération, motivation et satisfaction au travail. Paris : Économica.

ROUSSEL P. (2000). "Salaire individualisé - rémunération au mérite : impasse ou avenir? ». In J.-M. Perreti, P. Roussel (dir.), Les rémunérations. Politiques et pratiques pour les années 2000. Paris: Vuibert.

SYLVIA R. D. \& HUTCHINSON T. (1985). « What makes Ms. Johnson teach? A study of teacher motivation ». Human relations, vol. $38, \mathrm{n}^{\circ} 9$, p. $841-856$.

TIROLE J. (1992). «Collusion and the theory of organizations ». In J.-J. Laffont, Advances in economic theory: proceedings of the sixth world congress of the econometric society. Cambridge : Cambridge university Press, p. 151-206.

VROOM V. H. (1964). Work and motivation. New-York : John Wiley \& Sons.

WEST A. \& PENNELL H. (2005). « Market-oriented reforms and "high stakes" testing: incentives and consequences ". Cahiers de la recherche sur l'éducation et les savoirs, hors-série $n^{\circ} 1$.

WRIGHT S. P., HORN S. P. \& SANDERS W. L. (1997). «Teacher and classroom context effects on student achievement ". Journal of personnel evaluation in education, $n^{\circ} 11$, p. $57-67$ 